\title{
Imaging VISAR diagnostic for the National Ignition Facility (NIF)
}

R. M. Malone, J. R. Bower, D. K. Bradley, G. A. Capelle, J. R. Celeste, P. M Celliers, G. W. Collins, M. J. Eckart, J. H. Eggert, B. C. Frogget, R. L. Guyton, D. G. Hicks, M. I. Kaufman, B. J. MacGowan, S. Montelongo, E. W. Ng, R. B. Robinson, T. W. Tunnell, P. W. Watts, P. G. Zapata

September 16, 2004

SPIE High-Speed Photography and Photonics Conference Alexandria, VA, United States

September 19, 2004 through September 24, 2004 
This document was prepared as an account of work sponsored by an agency of the United States Government. Neither the United States Government nor the University of California nor any of their employees, makes any warranty, express or implied, or assumes any legal liability or responsibility for the accuracy, completeness, or usefulness of any information, apparatus, product, or process disclosed, or represents that its use would not infringe privately owned rights. Reference herein to any specific commercial product, process, or service by trade name, trademark, manufacturer, or otherwise, does not necessarily constitute or imply its endorsement, recommendation, or favoring by the United States Government or the University of California. The views and opinions of authors expressed herein do not necessarily state or reflect those of the United States Government or the University of California, and shall not be used for advertising or product endorsement purposes. 


\title{
Imaging VISAR diagnostic for the National Ignition Facility (NIF)
}

\author{
Robert M. Malone $^{*}$, John R. Bower ${ }^{\mathrm{b}}$, David K. Bradley ${ }^{\mathrm{b}}$, Gene A. Capelle ${ }^{\mathrm{c}}$, John R. Celeste ${ }^{\mathrm{b}}$, Peter \\ M. Celliers ${ }^{\mathrm{b}}$, Gilbert W. Collins ${ }^{\mathrm{b}}$, Mark J. Eckart ${ }^{\mathrm{b}}$, Jon H. Eggert ${ }^{\mathrm{b}}$, Brent C. Frogget ${ }^{\mathrm{a}}$, Robert L. \\ Guyton $^{\mathrm{d}}$, Damien G. Hicks ${ }^{\mathrm{b}}$, Morris I. Kaufman ${ }^{\mathrm{a}}$, Brian J. MacGowan ${ }^{\mathrm{b}}$, Sam Montelongo ${ }^{\mathrm{b}}$, Edmund \\ W. Ng ${ }^{\mathrm{b}}$, Ronald B. Robinson ${ }^{\mathrm{b}}$, Tom W. Tunnell ${ }^{\mathrm{a}}$, Phillip W. Watts ${ }^{\mathrm{d}}$, Paul G. Zapata ${ }^{\mathrm{d}}$ \\ ${ }^{\mathrm{a}}$ Bechtel Nevada, PO Box 809, Los Alamos, NM 87544; ${ }^{\mathrm{b}}$ Lawrence Livermore National \\ Laboratory, PO Box 808, Livermore, CA 94551; ${ }^{\circ}$ Bechtel Nevada, 5540 Ekwill St., Santa Barbara,

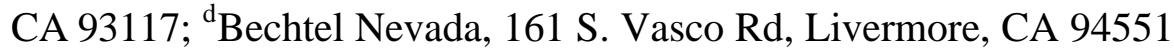

\begin{abstract}
ABTRACT
The National Ignition Facility (NIF) requires diagnostics to analyze high-energy density physics experiments. A VISAR (Velocity Interferometry System for Any Reflector) diagnostic has been designed to measure shock velocities, shock breakout times, and shock emission of targets with sizes from 1 to $5 \mathrm{~mm}$. An 8-inch-diameter fused silica triplet lens collects light at $\mathrm{f} / 3$ inside the 30 -foot-diameter vacuum chamber. The optical relay sends the image out an equatorial port, through a 2-inch-thick vacuum window, and into two interferometers. A $60-\mathrm{kW}$ VISAR probe laser operates at $659.5 \mathrm{~nm}$ with variable pulse width. Special coatings on the mirrors and cutoff filters are used to reject the NIF drive laser wavelengths and to pass a band of wavelengths for VISAR, passive shock breakout light, or thermal imaging light (bypassing the interferometers). The first triplet can be no closer than $500 \mathrm{~mm}$ from the target chamber center and is protected from debris by a blast window that is replaced after every event. The front end of the optical relay can be temporarily removed from the equatorial port, allowing other experimenters to use that port. A unique resolution pattern has been designed to validate the VISAR diagnostic before each use. All optical lenses are on kinematic mounts so that the pointing accuracy of the optical axis can be checked. Seven CCD cameras monitor the diagnostic alignment.
\end{abstract}

Keywords: VISAR, interferometer, streak camera , optical relay, National Ignition Facility (NIF)

\section{INTRODUCTION}

VISAR $^{1,2}$ (Velocity Interferometry System for Any Reflector) measures the velocity of a moving surface by recording its Doppler wavelength shift. The NIF (National Ignition Facility) VISAR diagnostic will be the primary diagnostic for timing the shocks induced into an ignition capsule. Fig. 1 shows the location for an imaging VISAR system that has been fielded to collect images from inside a 33-foot-diameter vacuum target chamber at NIF. ${ }^{3,4}$ Four drive laser beams (not shown) enter into the NIF target chamber through one of the largest ports at the bottom of the chamber and are focused onto a sample target. VISAR utilizes one of the equatorial

DOE/NV/11718—931. This work was supported by the U.S. Department of Energy, National Nuclear Security Administration Nevada Site Office, under Contract No. DE-AC08-96NV11718.

*malonerm@nv.doe.gov; phone 1505 663-2014; fax 1505 663-2003

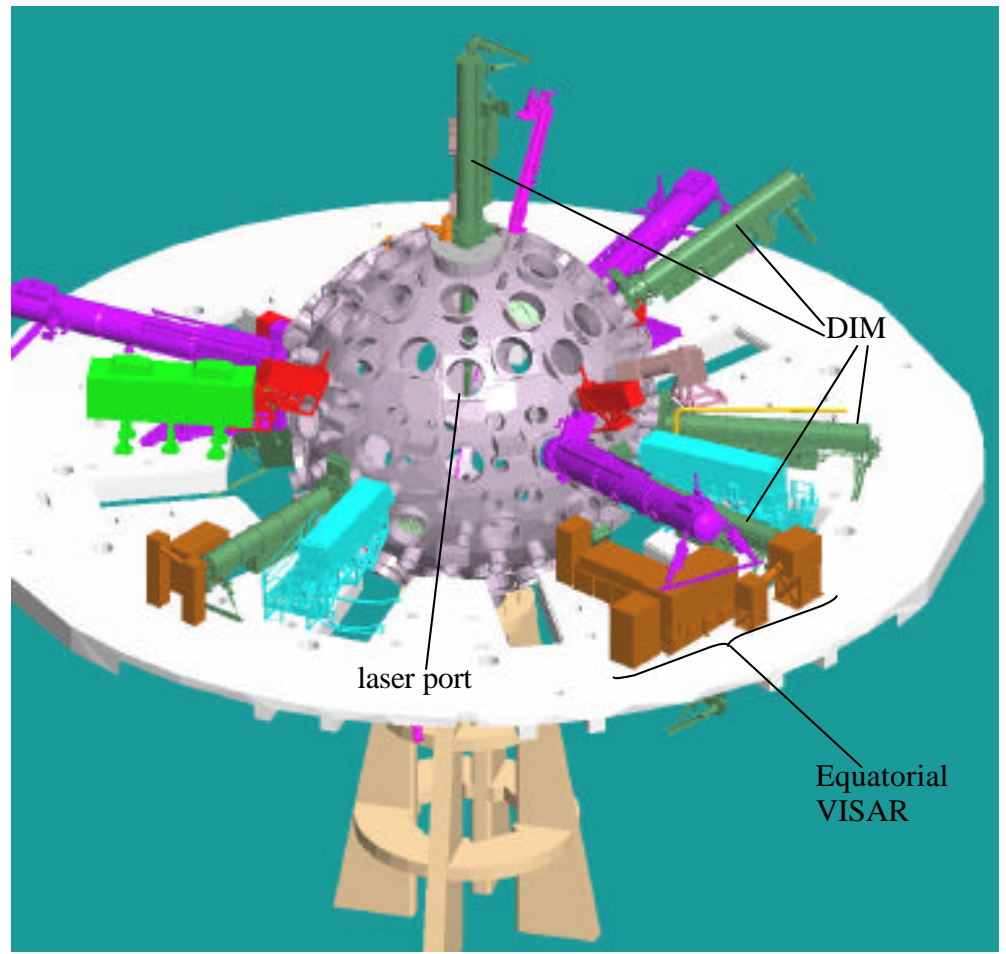

Fig. 1. NIF target chamber 
ports of this vacuum chamber. The Diagnostic Instrumentation Manipulator (DIM) used by VISAR has been designed for multiple users. The VISAR DIM cart hardware was designed to be compatible with existing DIM hardware designs.

VISAR images will show stronger shocks overtaking weaker ones, which is the basis of the plan for setting the pulse shape for the NIF drive lasers. ${ }^{5,6}$ A VISAR looking into liquid deuterium very accurately measures the speed of an approaching shock; the cold liquid is transparent, while the shocked deuterium is a highly reflective metal.

Fig. 2 shows the layout for this imaging VISAR, which routes the optical axis around a facility support pillar. A protective laser curtain encloses this diagnostic. Optics and mounting hardware inside the DIM are removable. The L3/M1 and M2 boxes, pinned to the floor, are detachable so that other diagnostics may use this same DIM location. The interferometer and laser tables are stationary. All enclosures for the DIM vacuum gate valve, DIM vacuum window, L3/M1 box, M2 box, interferometer table, and the probe laser table have safety interlock switches.

The DIM uses motor-controlled bipod legs for beam steering. This allows the imaging system to look anywhere within a $4 \times 4 \times 4 \mathrm{~cm}$ volume at the Target Chamber Center (TCC). Mirror M1 is oversized to allow for this steering;

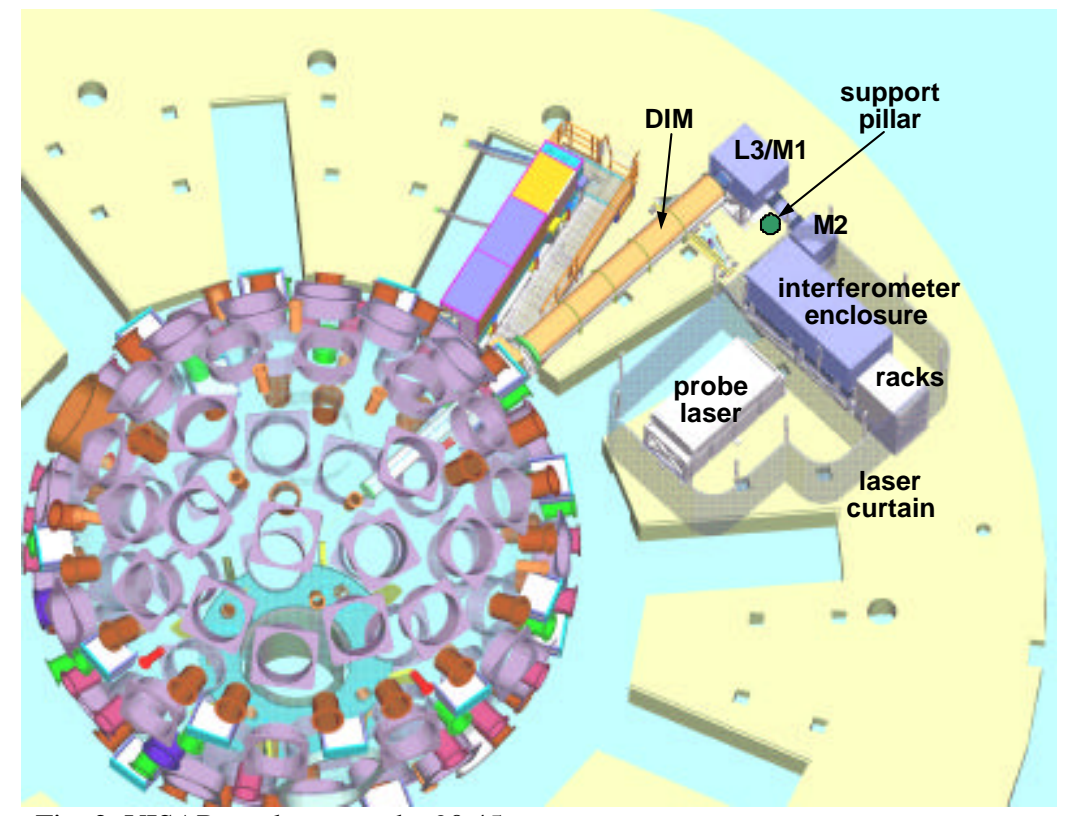

Fig. 2. VISAR work area at the 90,45 port however, doublet lens L3 must be shifted 10 inches to clear the correct optical axis. M1 has two tilting actuators under remote control. L3 has two tilt and two translation actuators under remote control. M2 is 20 inches lower in elevation than M1. M2 has two remote tilting actuators and is used to set the optical beam height for the interferometer table.

Laser operation and streak camera data recording are performed locally. The NIF facility control room (not shown) can also configure, arm, and collect data.

\section{OPTICAL DESIGN}

The optical design must be flexible to allow for changes in the placement of mirror boxes and the interferometer table. Because of our desire to collect light at $\mathrm{f} / 3$ to maximize light collected after shock breakout, and in consideration of the 500-mm stay-out zone at the TCC, the first fused silica triplet ens is 8 inches in diameter. Diffraction-limited resolution (down to a few microns) for 1 - to 5 -mm objects is required at a distance of 75 feet away. Image rotation at the streak camera slit is required. To maximize the light collected after shock breakout when that the shock surface becomes curved, it is desirable to illuminate the object with $\mathrm{f} / 3$ light from the probe laser.

The light from the TCC is collected by a triplet lens (L1) and exits the DIM through a 2-inch-thick vacuum window (see Fig. 3). An intermediate image is formed inside the DIM and is picked up by a doublet lens (L3) placed in front of the first turning mirror (M1). A second turning mirror (M2) lowers the optical path to the interferometer table.

To minimize damage to expensive optical components, mirrors M1 and M2 have special coatings that do not reflect 1053-nm, 527-nm, or 351-nm NIF drive laser wavelengths. All optical elements, including mirrors, from the TCC to beam splitter BS1 (on the interferometer table) are fused silica, to minimize radiation darkening caused by the intense $\mathrm{X}$-rays of some NIF experiments. Narrow-band filters $(3 \mathrm{~nm})$ and cutoff filters are placed behind dove prisms, where 


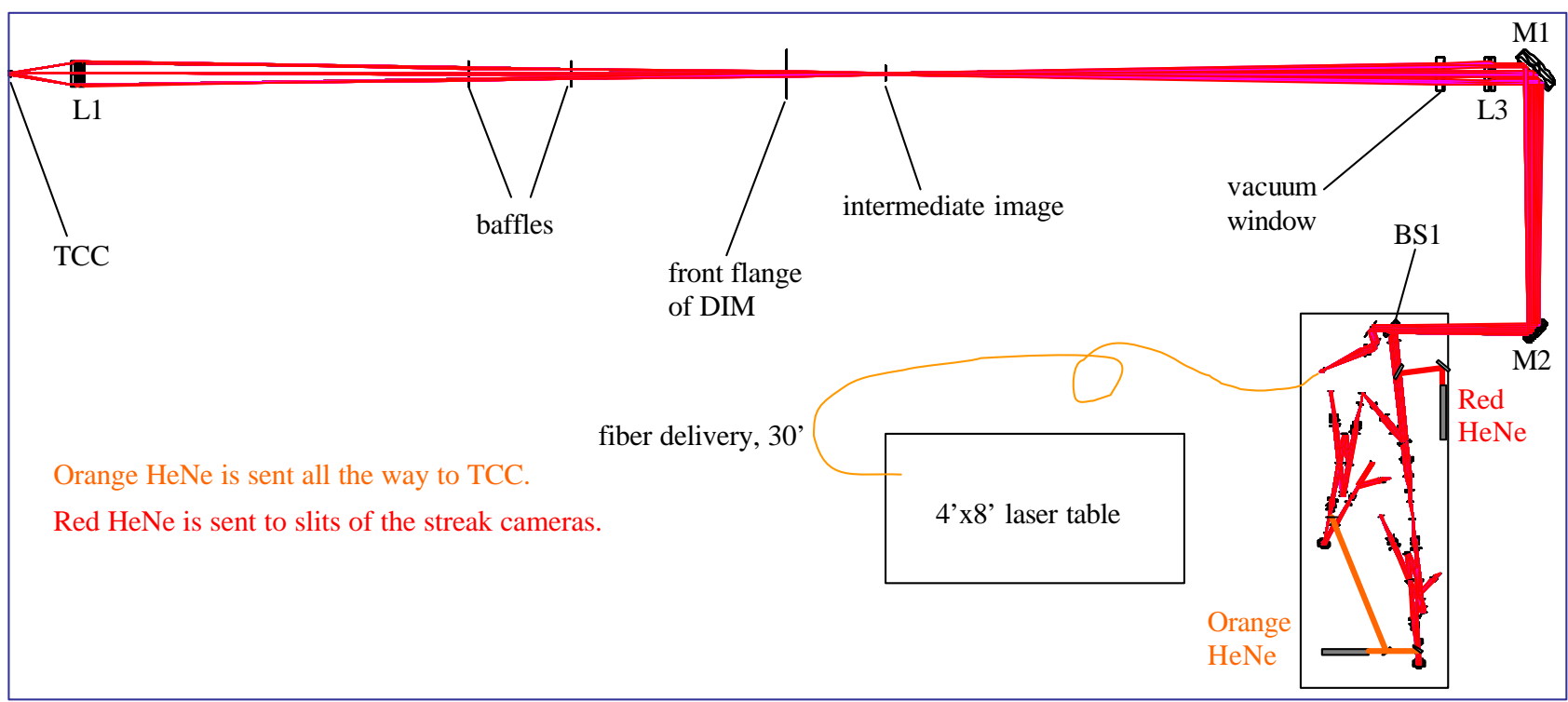

Fig. 3. Labeling of the equatorial optical relay system shown in Fig. 2.

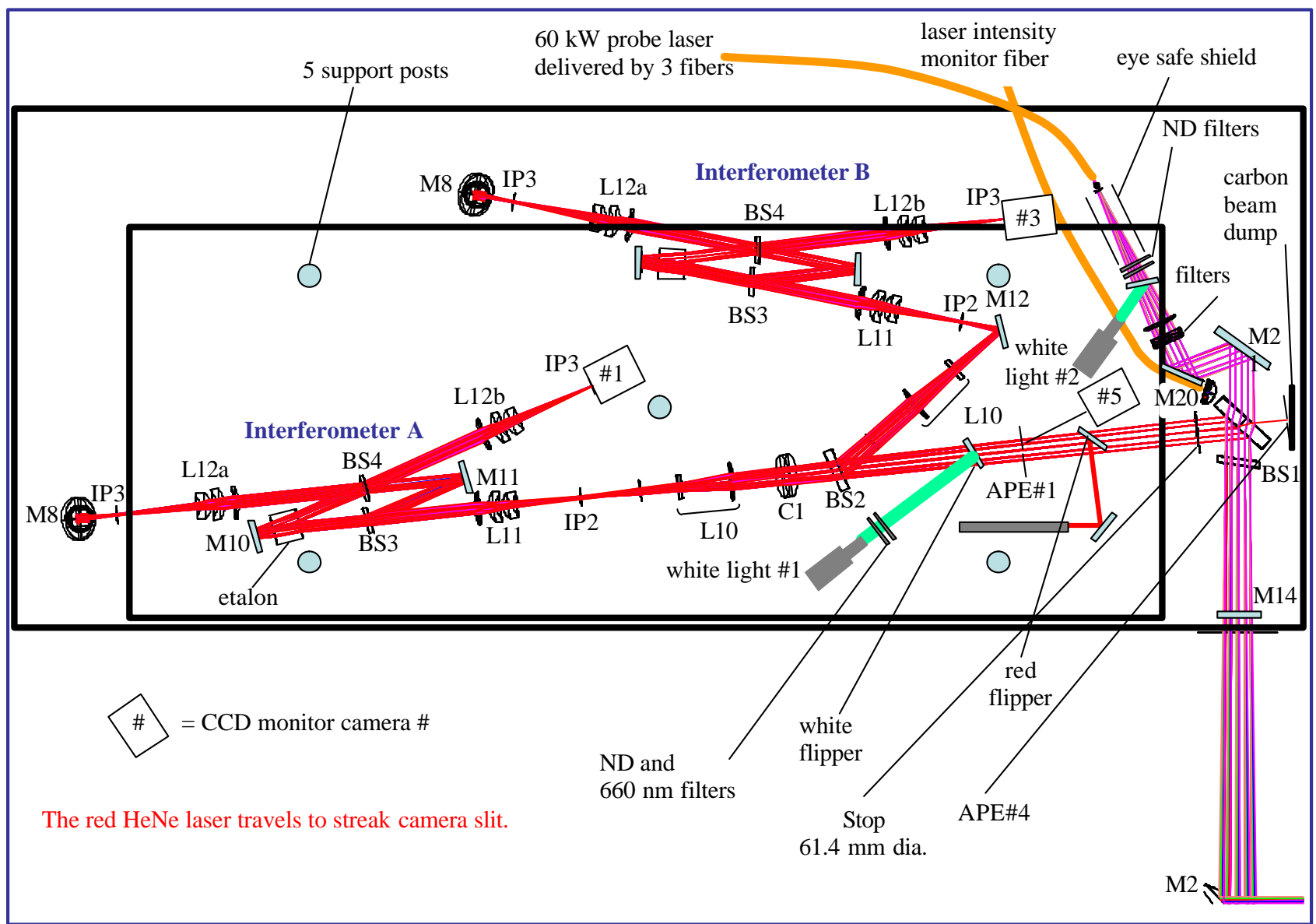

Fig. 4. Optical ray tracing through lower level of interferometer table. 
the relayed light is almost collimated. This adds protection to the streak camera data from the NIF drive lasers. A wider, 70-nm filter in the laser launch section also prevents NIF drive lasers from focusing onto the fiber end tip.

Light from a $60-\mathrm{kW}$ probe laser (see Section 4) is delivered by three large-core, 1-mm-diameter optical fibers. This light enters the optical system through a 50/50 beam splitter, BS1. Half of the probe's laser power is lost to a carbon beam dump, which prevents unshifted probe laser light from entering the interferometer. Light from the TCC also uses this beam splitter. Half of the VISAR return signal is lost at this beam splitter.

Because of space constraints, the two interferometers are located on the lower shelf of the interferometer table (Fig. 4), while the recording streak cameras occupy the upper level of the table (Figs. 5 and 6). At this table, beam splitter BS2 splits the light into two separate interferometers. Each interferometer can have its own etalon (different velocity range) and image rotation, using a 37-mm aperture dove prism (Fig.6). A 1-1 optical relay is placed after the interferometer (image plane IP3) and relays the image from the lower to the upper breadboard level (image plane IP4). The dove prism is placed in the waist of the collimated section of this $1-1$ optical relay.

The interferometer uses a delay etalon in one of its legs, so that at the recombining beam splitter, fringe shifts will move as the Doppler-shifted wavelength changes. Light is collimated at this beam splitter. The white light \#1 is used to achieve interference fringes without the etalon inside the interferometer. After centering the white light fringes on CAM2 and CAM4, the etalon is inserted in front of mirror M10. M10 is then translated on a precision stage to set the back-off distance that the etalon requires.

The original optical design used pairs of Nikkor lenses to relay image plane IP4 (Fig. 6) to the slit of the streak camera. These lenses were mated together with a flange that secured them into a dual-angle tilt mount. By changing the lenses' focal length ratio, different magnification could be applied to the image presented to the camera slit. For example, to image a 1 -mm object size to the slit, we chose a $50-\mathrm{mm}$ lens followed by a $180-\mathrm{mm}$ lens. Thus, we could record object sizes ranging from 1 to $5 \mathrm{~mm}$ in diameter. However, the focusing and aligning of this Nikkor pair proved to be time-

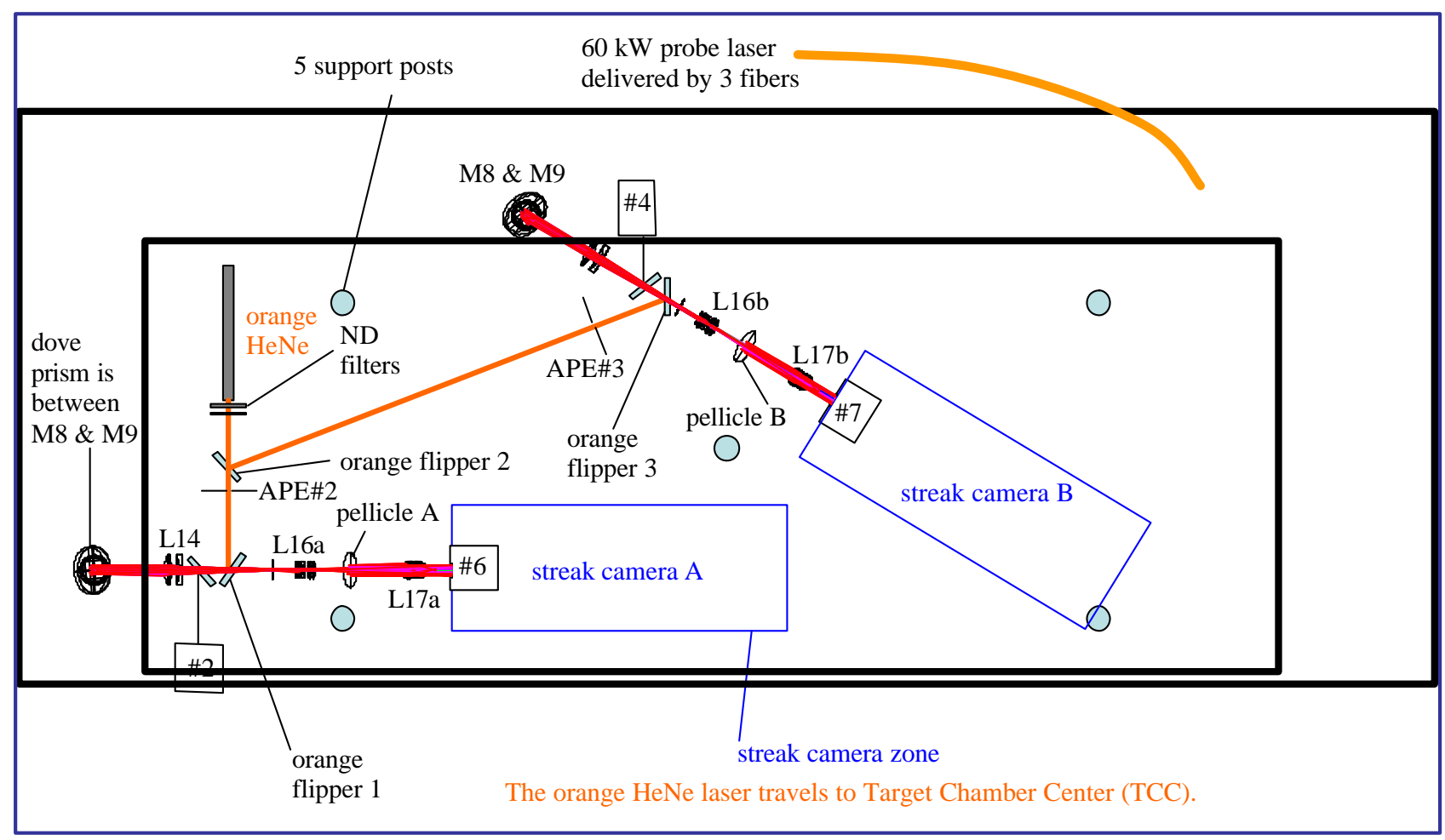

Fig. 5. Optical ray tracing through upper level of interferometer table. Using orange flipper 2, the orange laser is sent either through interferometer A or B. 


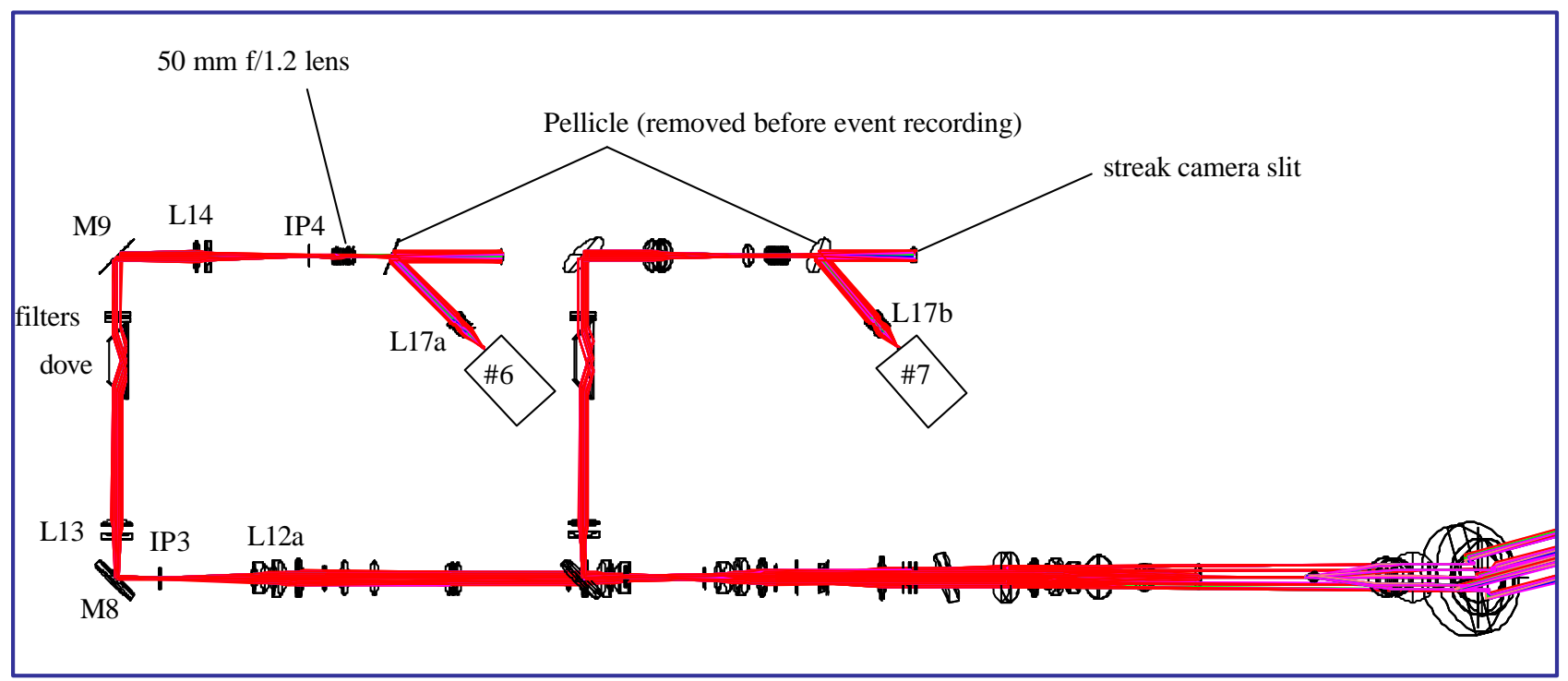

Fig. 6. Use of cameras \#6 and \#7 allows for magnification calibrations and exact centering of the images onto the streak camera slits. Light is collimated through the dove prisms and filters.

consuming. In addition, we wished to reduce the number of optical elements in front of this slit. Nikkor lenses have general broadband AR coatings. At $660 \mathrm{~nm}$, internal reflection losses would reduce the fringe contrast.

The solution was to use a single 50-mm Nikkor lens (L16) to relay the image from IP4 to the streak camera slit. To maintain the optical axis, two different color lasers are used: a red HeNe at $633 \mathrm{~nm}$ and an orange $\mathrm{HeNe}$ at $612 \mathrm{~nm}$. However, these two alignment lasers do not focus the image planes at their correct locations. A 660-nm CW diode laser, which is made collinear with the probe laser inside the laser enclosure, is used for focusing the optics with the correct wavelength. White light sources with narrow-band filters equalize the optical path differences of the interferometer legs. These white light sources are used to set up the white light fringes necessary to calibrate the velocity being recorded. Because it poses an eye hazard and will only fire at $\mathrm{Hz}$ repetition rates, the probe laser is only used to record streak camera data.

The streak camera, mounted onto ball bearing guide rails, could easily be positioned. However, depending on the exact position of the 50-mm lens, magnification could not be calibrated. As a solution, a 50/50 pellicle was mounted onto a kinematic plate and placed between the 50-mm lens and the slit. Light from this pellicle was picked up by a macro lens and viewed by a CCD camera (note CAM6 and CAM7 positions in Fig. 6). For purposes of this optical layout, to direct all the field points through macro lens L17 and into CAM6 and CAM7, a curved mirror (620-mm focal length) was temporally substituted at the streak camera slit plane (Fig. 6). Thus, this curved mirror acted as a field lens. The slit, anodized black, was diffuse enough to allow equal reflection of all field points into CAM6 and CAM7. Consequently, when using CAM6 and CAM7 with the white light \#1, we could view the focusing of the image plane IP2 reticules. White light \#1 or \#2 was introduced into the optical system using flipper mirrors, and images of the magnifications were archived using a frame grabber. When the 660-nm CW light was used, CAM6 and CAM7 allowed us to set the fringes perpendicular to the slits. Before recording data with the probe laser, the IP2 reticules, flip mirrors, apertures, and the pellicles were all removed.

Image plane IP2 is assigned as the reference plane. Mirror M14 is used when $660-\mathrm{nm}$ CW diode light or the probe laser is needed but there is no access through the DIM to TCC. M14 is mounted onto a kinematic base and must be removed when light is sent into the M2 mirror box (Fig. 4). Either light source provides images to the monitoring CCD cameras. Most of the optical alignment occurs without access to the DIM. When access to the target at TCC is available, L1 is used to focus and overlap the object at the established IP2 plane. Lens L10 can also be used for focus. For fine focus of the target at the TCC, white light \#2 can also be used to eliminate the speckle in the image that laser illumination of the target produces. 
Fig. 6 shows the dove prisms placed between identical pairs of collimating relay lenses (L13 and L14). These lenses pass light from intermediate image planes IP3 to IP4. The doves are placed at the waist of the collimated light. Rotating these doves is performed while viewing the TV monitors and viewing the red and orange laser spots at APE\#1 and APE\#2. Such rotation causes the image to shift if the dove is not precisely aligned. Because the light is collimated through the dove, one can correct an image shift (as viewed on the monitor) by translating the rotation stage that the dove prism is mounted inside of. Translating the rotation stage and tilting the dove prism is iterated upon until the red and orange laser beams are collinear and passing through APE\#1 and APE \#2.

The comb and FIDU trigger optical fibers are glued into a 90-degree bent stainless-steel tube and mounted on both sides of the streak camera slit. Allowing for these fibers, the available slit width for the line image is $19 \mathrm{~mm}$. When mirror M14 (located at the port leading to the M2 mirror box in Fig. 4) is used for dry runs, a time shift to the streak camera trigger is performed. After removing M14, a final dry run with the comb, FIDU, and static fringes are performed less than 15 minutes before a NIF event.

\section{ALIGNMENT SYSTEM}

Fig. 4 illustrates the entrance of a red HeNe alignment laser into the optical axis by way of a flip mirror. This laser is centered on the slit of the streak camera and also on the output coupler of an orange $\mathrm{HeNe}$ alignment laser (Fig. 5). The orange $\mathrm{HeNe}$ laser also uses a flip mirror to enter the optical axis and is centered on the output coupler of the red $\mathrm{HeNe}$ alignment laser as well as aperture cards APE\#1 and APE \#4 (Fig. 4). APE\#1 and APE\#4 maintain the orange laser axis that ultimately terminates at the TCC. APE\#4 is permanently mounted. APE\#1 receives red retro-reflections off lenses and receives orange dots steered by these same lenses. APE\#1, mounted on a kinematic plate, is removed when the streak cameras record images. A paper reticule, with circles ranging from 5 to $25 \mathrm{~mm}$, is mounted on the back side of APE\#1; in this way CAM5 (Fig. 4) can view the back side of APE\#1 and record the tilt misalignment of the target placed at the TCC. Great care is taken to keep the red and orange lasers collinear to each other.

Fig. 5 shows flipper mirrors to redirect the images onto CCD cameras (CAM2 and CAM4) that the streak cameras' slit would see. These cameras help set the orientation of the fringes, set fringe spacing, and show fringe contrast. By placing Air Force resolution targets at image planes IP2 located before the interferometers, these cameras can document magnification. Images can then be fed into a frame grabber that archives alignment performances.

Maintaining stability over a very long optical path length is challenging. The shear number of optical elements make alignment complex: alignment checks must be done on more than 60 optical components (some having seven glass elements), compound angles exist, and numerous VISAR operators are expected to use this diagnostic with different setups (etalon thickness, magnifications).

The optical axis is established with all lenses removed. The lenses are all mounted onto commercial kinematic bases. After clearing all lenses away from the optical axis, we test each lens group individually for centering and tilt. An operator using only one laser beam could incorrectly alter a lens decenter by tilting it. A single laser would not suffice because one can compensate for a lens tilt (either angle) by a decenter (shift in either the X or Y direction) and not satisfy the alignment requirements. By using two differently colored lasers propagating in opposite directions, precise alignment of each lens can be achieved. The optical elements have a Vcoat for $660 \mathrm{~nm}$ and provide reasonable reflections at 612- and 633-nm wavelengths of the orange and red lasers. Beam steering and retro-reflections are viewed on movable apertures using both differently colored alignment lasers. Each laser beam produces both forward steering spots and retro-reflection spots centered onto these aperture cards.

Using a telescope or an autocollimator for alignment would usually limit the viewing of alignment errors to the operator. Alignment aids would have to be repeatedly repositioned to cover the optics located on both levels inside the interferometer enclosure. However, using aperture cards to display the two-colored laser alignment errors lets multiple users sample/probe for possible adjustments. Operators have found this method uncomplicated.

If the IP2 (resolution pattern) position on interferometer B differs from interferometer A, then there will be a slight difference in magnification at the two IP4 image planes. In addition, we are using 50-mm Nikkor lenses to relay IP4 
images onto the slits. If the A or B positions of this Nikkor lens are not the same, then a magnification difference will also exist. Magnification at the streak cameras should be the same, but this is difficult to achieve. However, magnifications can be calculated by using the pellicles, CAM6, and CAM7. One records the relayed IP2 image of a resolution pattern at the slit as the magnification reference. One can also put a resolution target at TCC and overlay this with the IP2 resolution pattern; however, gaining access to TCC is usually only achieved at the start of a campaign series.

Fig. 7 shows our optical system alignment capability. The 660-nm CW laser diode was sent to an Air Force resolution pattern placed at TCC. Light reflects off the bar patterns as white patterns. The reflected light provides a back light to another Air Force resolution pattern placed at the intermediate image plane IP2 (dark patterns), located just in front of the interferometer. By overlapping these two patterns, we can archive the image centering, rotation, and magnification. The monitor CCD cameras use a frame grabber located in the diagnostic computer.

A newly designed resolution pattern appears in Fig 8. In addition to the positive and negative Air Force resolution patterns, there exist other patterns to qualify the imaging performance. One pattern (B) has a series of dots ranging from 2.5 to $30 \mu \mathrm{m}$, to analyze loss of resolution due to laser speckle. Pattern (B) also has line widths from 5 to $25 \mu \mathrm{m}$. The

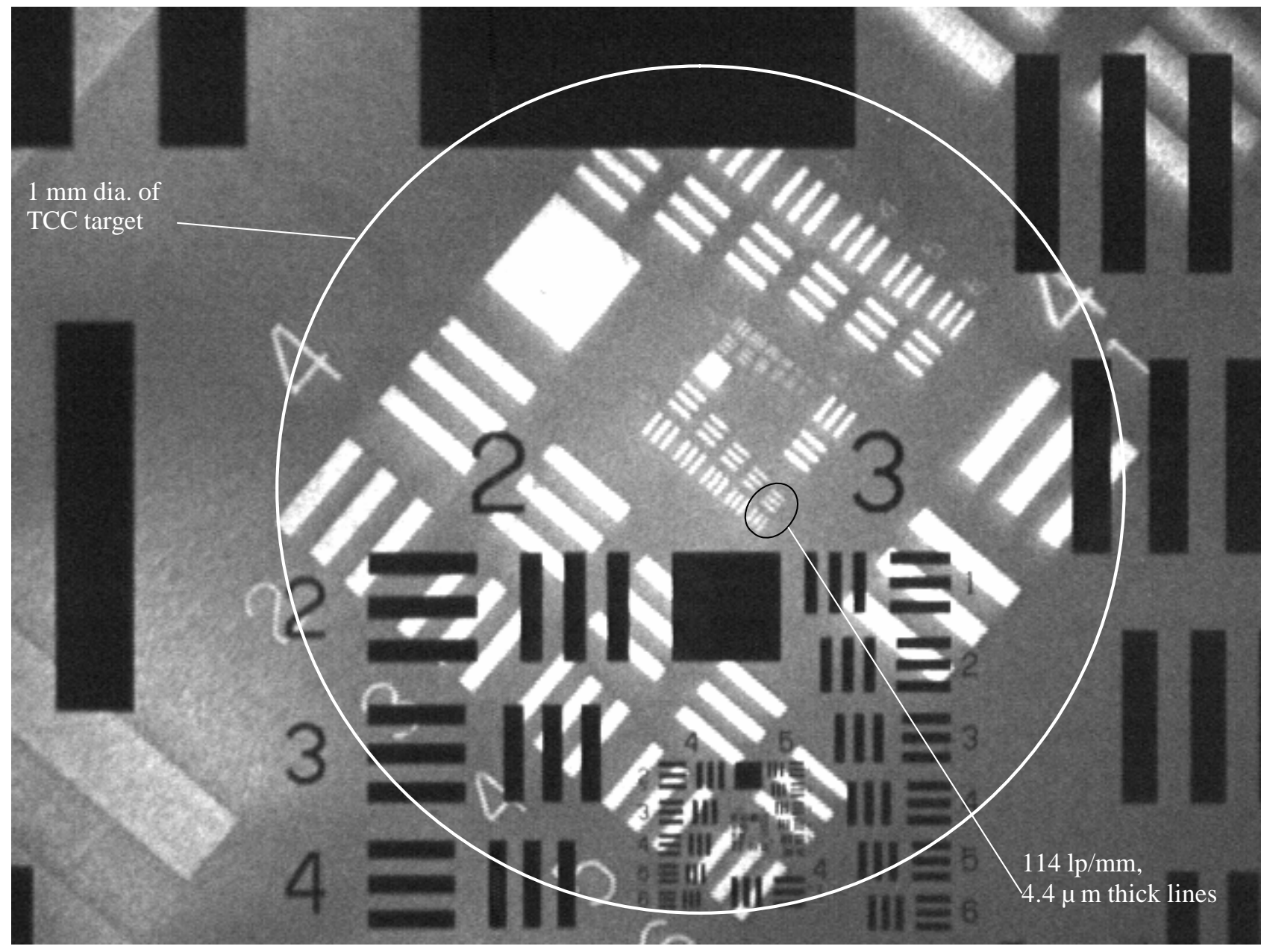

Fig. 7. Air Force target @ TCC illuminated by 660-nm CW laser diode (reflection mode) superimposed with Air Force target at IP2 (transmission mode). Dove prisms will provide correct image rotation onto the streak camera slits (not done for this archived image). Image plane centers have been separated to show resolution differences. 


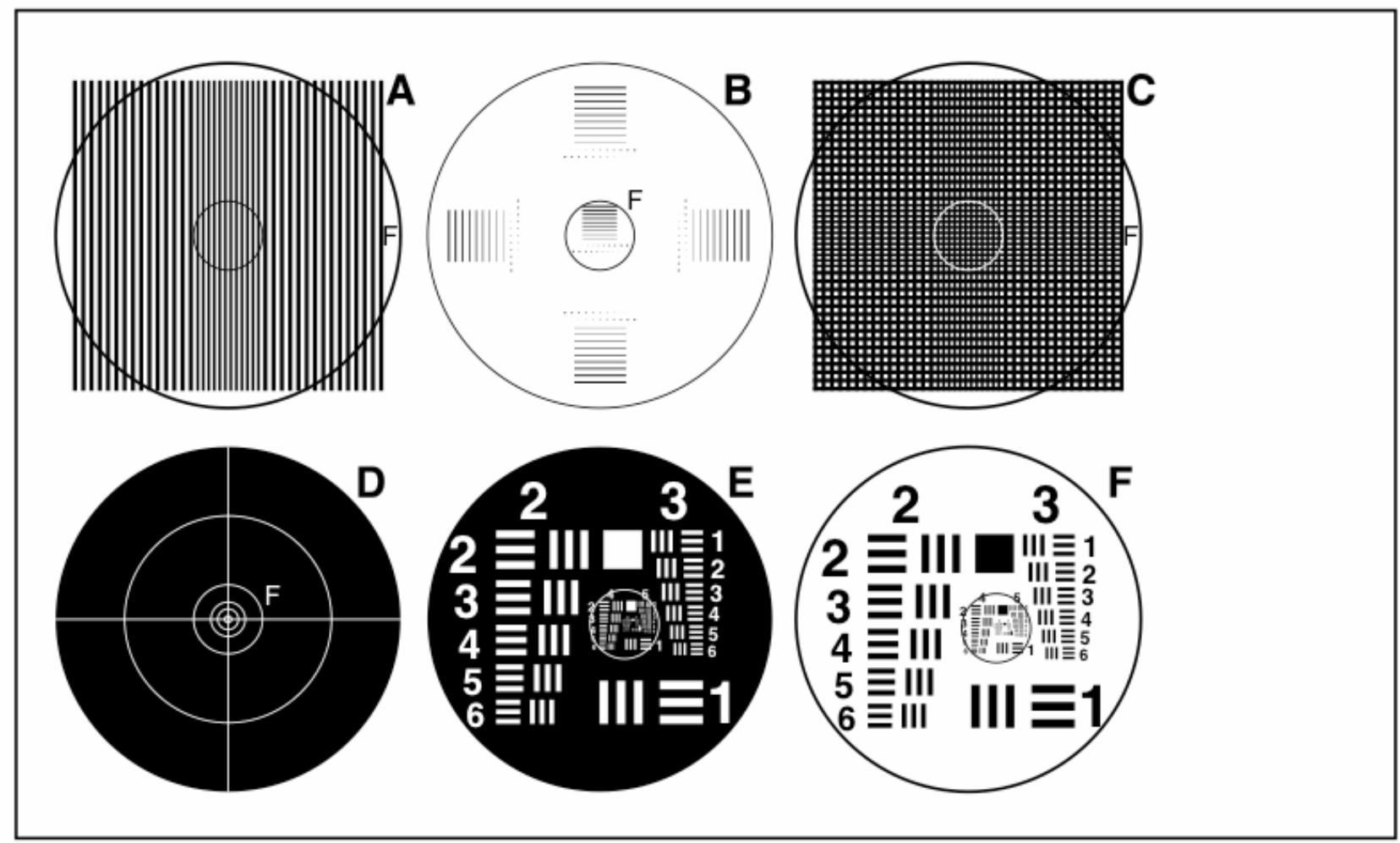

Fig. 8. New VISAR alignment glass plate target measuring $6 \mathrm{~mm} \times 20 \mathrm{~mm}$.

grid pattern (C) permits measurement of distortion. The reflector pattern (D) allows measurement of the dynamic range of the VISAR imaging system. Sufficient copies of this resolution plate exist to place in rotatable mounts at different intermediate image planes. This new resolution target will be used to qualify VISAR before each shot campaign.

\section{VISAR PROBE LASER SYSTEM}

The VISAR probe laser must illuminate the target before and during the shocked surface's motion. The probe laser wavelength had to be far enough away from the three NIF drive laser wavelengths (Fig. 9), and it had to have a narrow line width (single frequency) for VISAR operations. The $60-\mathrm{kW}$ probe laser used in this VISAR system is a doubled Nd:YAG laser operating at $659.5 \mathrm{~nm}$. The probe laser light originates from a 1319-nm CW seed laser and passes through a Pockels cell pulse shaper, which gives reasonably flat-topped output of $1.2 \mu \mathrm{s}$ in duration. If the Nd:YAG laser amplifiers are driven too hard, $1064 \mathrm{~nm}$ is difficult to suppress. The gain of the amplifiers had to be reduced and the number of laser amplifiers had to be increased from 7 to 14. Each of the seven laser heads contained two laser rods. Laser rods had to be two-layer AR-coated to prevent the 1064-nm wavelength from becoming parasitic. The laser was optimized at a sub-Hertz repetition rate, to retain alignment when fired only occasionally. Many high-speed detectors were added at selected control points to monitor laser performance.

Three 1-mm core delivery fibers transmit laser light to the interferometer table. Three fibers were chosen to minimize the risk of Brillouin scattering that could occur if too much laser light we re injected into a single fiber. A cube beam splitter system splits the laser light into three, 1-meter-long jumper fibers with 1-mm core diameters. An energy meter equalizes the light while adjusting half-wave plates. The light is then coupled into 30 -foot-long delivery fibers. With a flip mirror inside the laser table enclosure, either a $660-\mathrm{nm} \mathrm{CW}$ diode laser or the pulsed probe laser is allowed to enter into the delivery fibers. The 660-nm CW diode laser has variable power up to $200 \mathrm{~mW}$. 


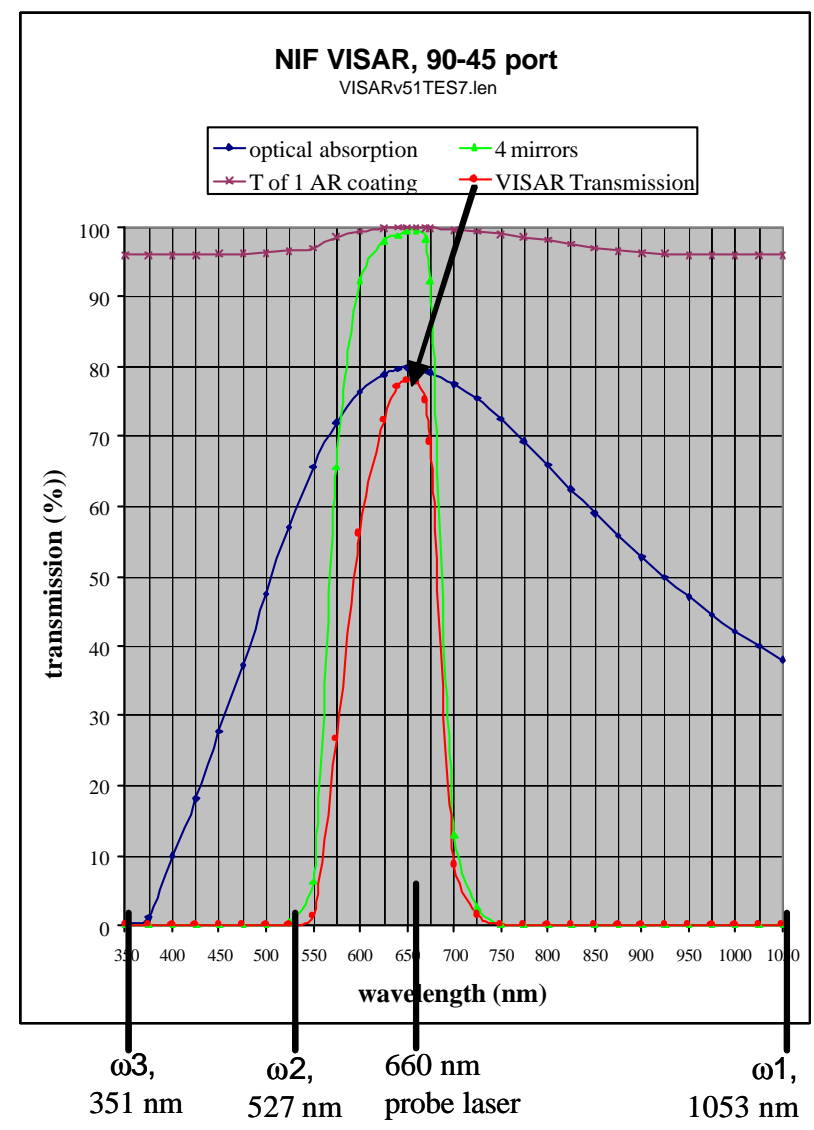

Fig. 9. Transmission curves for VISAR imaging. The 660-nm probe laser is positioned away from the NIF drive lasers.

The shape of the original pulse (1.2 $\mu \mathrm{s})$ is matched to the nonlinear gain of the amplifiers. When the pulse shaper turns off, acoustic ringing occurs in the Pockels cell. As a result, a series of weak after pulses, a factor of 1000x lower in intensity than the main pulse, is produced in the laser output. A second Pockels cell was added to the probe laser just after the second harmonic generator to block the tail of the pulse. The first version of this Pockels cell tail chopper had an average extinction ratio of only $15: 1$. We are awaiting delivery of a version with a higher extinction ratio.

\section{OPTOMECHANICAL ASSEMBLY}

The mechanical designs must be earthquake-tolerant. The laser and interferometer tables have earthquake restraints and the electrical rack is shock mounted. Excluding the laser system, more than 60 optical components exist. One component weighs 49 pounds
(M1). Weight of the L1 housing with its glass is 20 pounds. Another component has 7 optical elements (50-mm f/1.2 Nikkor lens). The total thickness of glass and mirrors is almost 3 feet.

Mounting gimbals for the L3 lens doublet, the M1 mirror, and the M2 mirror were designed and fabricated in-house. The optical elements were nonstandard sizes and commercial mounts would not satisfy design constraints. The L3 doublet lens (10.5inches) has to move 10 inches to clear the optical axis during target alignment operations. M1 and M2 mirror boxes need to be removed while any other DIM carts that use this equatorial port are being installed.

The DIM has a two-stage extension to deliver an instrument close to the TCC. All diagnostics use a standard extension tube with a precision traveling screw. Each target chamber diagnostic requires a unique package that travels inside this extension tube for placement. In Fig. 10, the VISAR diagnostic fully extends past the vacuum gate valve towards the TCC. Because of the two-stage extension method used to get L1 close to the TCC, it was difficult to mount other relay lenses inside the DIM. Therefore, we located the next relay lens (L3) just outside the large, 10.5-inchdiameter vacuum port window (Fig. 9). Crosshairs are mounted onto the L1 frame as an orange laser alignment aid.

The VISAR diagnostic cart that is inserted into the DIM is a two-section design, necessary to achieve a long extension towards TCC. The L1 triplet lens is mounted to the front of this DIM cart. Limit switches and hard stops guarantee that the VISAR cart will not overshoot and fall into the vacuum chamber. Fine focus of L1 is achieved by driving the precision extension drive screw.

Fig. 11 portrays the VISAR cart retracted outside the vacuum chamber and ready for servicing. An earlier design had a cart length of 17.5 feet, with difficult access for L1 lens removal. To improve the design, a field lens (L2) placed close to a baffle at the cart motor mounting plate was eliminated, and the diameters of the vacuum window, relay doublet lens L3, and mirror M1 were increased. After repositioning the cart baffles, no vignetting or loss of resolution resulted from these changes. Now the L1 doublet lens and its blast window have easy access through a side panel of the DIM. The blast window may have to be replaced after every event if damage is seen upon inspection. 


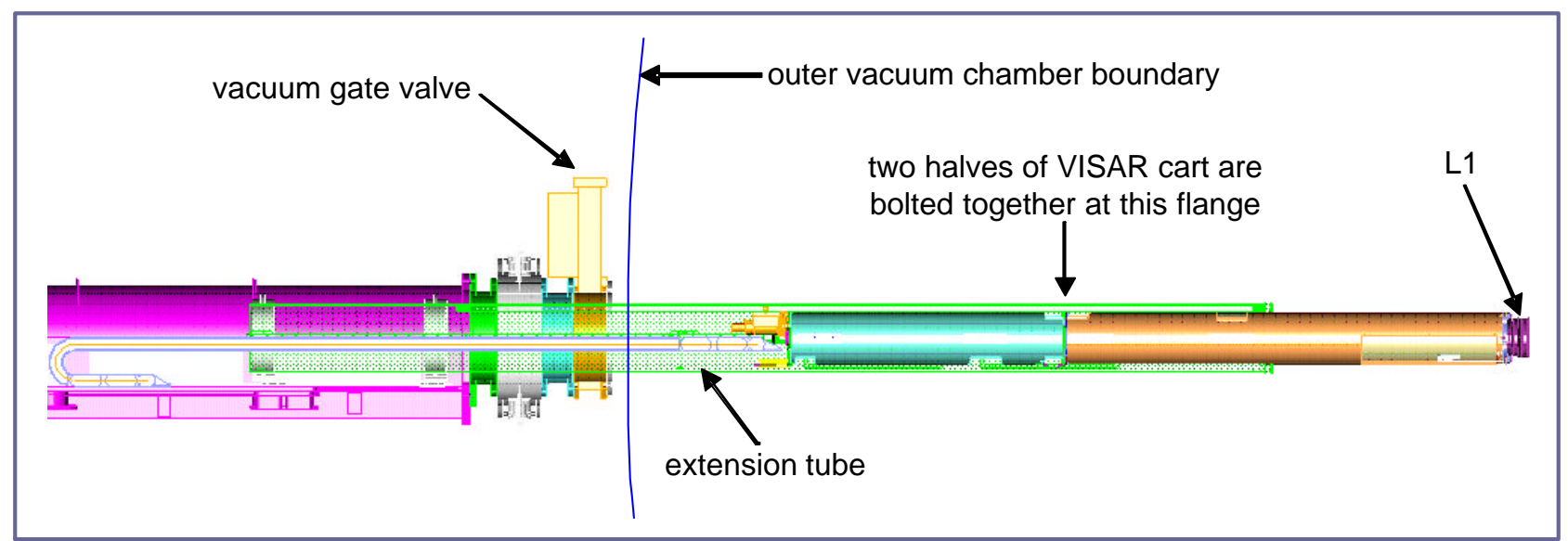

Fig. 10. VISAR diagnostic cart fully extended. The blast window in front of the L1 triplet lens is $503 \mathrm{~nm}$ from the TCC. Limit switches and hard stops limit the movement of the extension tube and the VISAR cart.

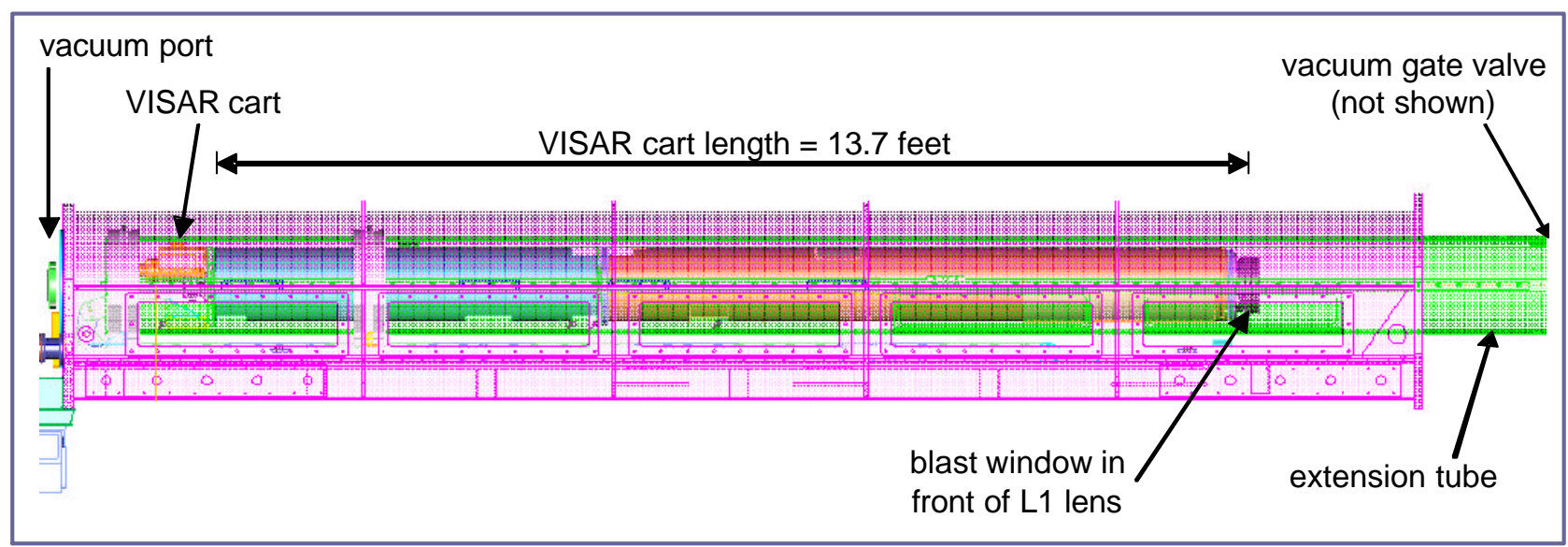

Fig. 11. VISAR diagnostic cart in retracted position. Blast window can be swapped out through vacuum port panel.

\section{INTERFEROMETER IMPROVEMENTS}

The initial interferometer optical components are mounted directly to the $4 \times 8$-foot optical table using posts and post holders. However, this proved inadequate for long-term stability of interference fringes, as the thermal environment of the enclosure changed over several hours. Aluminum kinematic plates are being retrofitted to both interferometers, as shown in Fig.12. The bottom plate is bolted to the surface of the interferometer table, and the upper plate rests on the bottom plate at three kinematic points. The optical components that comprise the interferometer rest on the upper plate. Now no warping of the interferometer table can be transferred to the interferometer optics. Side-by-side fringe drift measurements on another test bed interferometer table have been made between the original interferometer layouts versus the kinematic plate interferometer layout. Data show that the original layout was much more susceptible to both temperature and vibrations.

The interferometer optics contain two beam splitters, two mirrors, and one etalon. One of the mirrors moves along a precision translation stage. The beam splitters are orientated such that each leg of the interferometer passes through one thickness of a beam splitter before the interference pattern is generated. Identical relay lenses are placed before and after this kinematic plate, which is designed to be removed from the interferometer table for calibration. 


\section{SUMMARY}

Some of our optics must work inside vacuum systems and in high-radiation environments. The flexible optical relay system allowed for air path length changes during the fielding. After installation of the VISAR imaging system, we were able to maintain diffraction-limited optical performance. For a 5-mm field of view, 1 part in 700 was resolved at the streak camera slit. For a 1-mm field of view, 1 part in 250 was resolved.

We designed the ability to quickly remove all optics from beam path for alignment checks. The two-colored laser alignment scheme is accurate and compatible with optical systems having a large number of components. The flexible optical relay system can accommodate a future thermal imaging system that is under development. Using this design, high-speed framing camera image recording can also be accommodated.

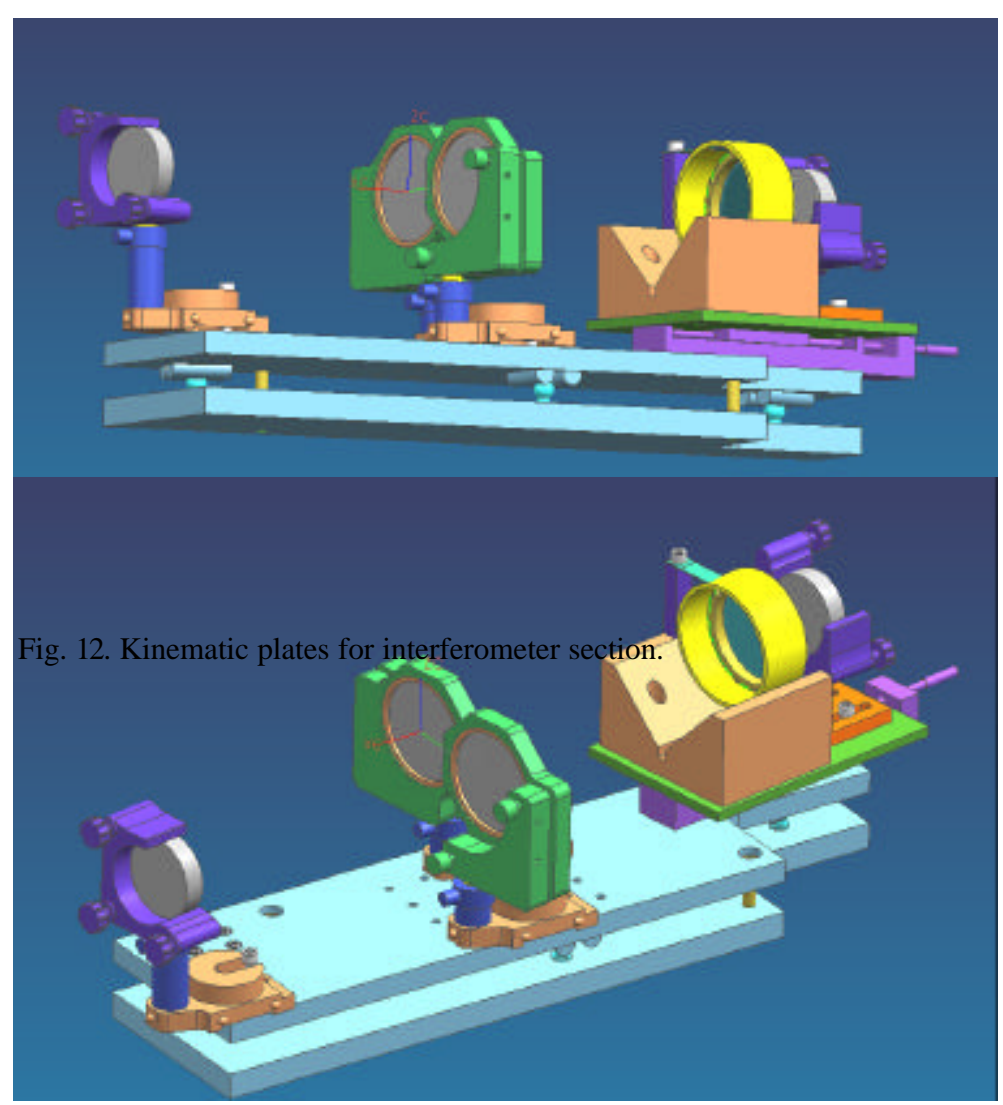

\section{ACKNOWLEDGMENTS}

The authors would like to thank the following Bechtel Nevada personnel for opto-mechanical support: Jim Daniel, Kevin McGillivray, Steve Slavin, Bill Skarda, and Aric Tibbitts. Peter Celliers of LLNL is the principal VISAR diagnostic physicist. Ed Ng of LLNL is the VISAR project manager. Brian MacGowan is the NIF target diagnostic manager.

This work was performed under the auspices of the U. S. Department of Energy by University of California, Lawrence Livermore National Laboratory under contract No. W-7405-Eng-48. 


\section{REFERENCES}

1. L. M. Barker and R. E. Hollenbach, "Laser interferometer for measuring high velocities of any reflecting surface," J. Appl. Phys. 43, 4669, 1972; L. M. Barker and K. W. Schuler, "Correction to the velocity-per-fringe relationship for the VISAR interferometer,” J. Appl. Phys. 45, 3692, 1974.

2. D. D. Bloomquist and S. A. Sheffield, "Optically recording interferometer for velocity measurements with sub nanosecond resolution,” J. Appl. Phys. 54, 1717, 1983.

3. R.M. Malone, B.C. Frogget, M.I. Kaufman, P.W. Watts, P.M. Bell, J.R. Celeste, T.L. Lee, "Design of an imaging VISAR diagnostic for the National Ignition Facility (NIF)," SPIE Proc. 5173, 26-37, 2003.

4. R.M. Malone, G.A. Capelle, B.C. Frogget, R.L. Guyton, M.I. Kaufman, G.A. Lare, T.W. Tunnell, P.W. Watts, J.R. Bower, J.R. Celeste, P.M. Celliers, T.L. Lee, B.J. MacGowan, S. Montelongo, E.W. Ng, T.L. Thomas, "Fielding of an imaging VISAR diagnostic at the National Ignition Facility (NIF)," SPIE Proc. 5523, 2004.

5. P.M. Celliers, G. W. Collins, L. B. Da Silva, D. M. Gold, R. C. Cauble, R. J. Wallace, M. E. Foord, and B. A. Hammel, "Shock-Induced Transformation of Liquid Deuterium into a Metallic Fluid," Phys. Rev. Lett. 84, 55645567 (2000).

6. D. H. Munro, P. M. Celliers, G. W. Collins, D. M. Gold, L. B. Da Silva, S. W. Haan, R. C. Cauble, B. A. Hammel, and W. W. Hsing, "Shock timing technique for the National Ignition Facility," Physics of Plasmas, 8, 2245 (2001). 